\title{
ORDER OF APPROXIMATION FOR SAMPLING KANTOROVICH OPERATORS
}

\author{
DANILO COSTARELLI AND GIANLUCA VINTI
}

\begin{abstract}
In this paper, we study the problem of the rate of approximation for the family of sampling Kantorovich operators in the uniform norm, for uniformly continuous and bounded functions belonging to Lipschitz classes (Zygmundtype classes), and for functions in Orlicz spaces. The general setting of Orlicz spaces allows us to directly deduce the results concerning the order of approximation in $L^{p}$-spaces, $1 \leq p<\infty$, very useful in applications to Signal Processing, in Zygmund spaces and in exponential spaces. Particular cases of the sampling Kantorovich series based on Fejér's kernel and B-spline kernels are studied in detail.
\end{abstract}

1. Introduction. In [4] the authors introduced the sampling Kantorovich operators and studied their convergence in the general setting of Orlicz spaces in the one-dimensional case. Later these results have been extended in [19] to the multivariate setting, in $[\mathbf{2 0}, \mathbf{3 9}]$ to the nonlinear case and in a more general context in $[\mathbf{7}, 40]$. Applications to civil engineering images have been studied in [18].

In this paper, we study the problem of the rate of approximation for the sampling Kantorovich operators in various settings. We consider the case of uniform approximation for uniformly continuous and bounded functions belonging to Lipschitz classes and the case of modular approximation for functions in Orlicz spaces. In this context, we will introduce Lipschitz classes of Zygmund-type which take into account the modular functional involved.

The sampling Kantorovich operators, considered here, are of the form

2010 AMS Mathematics subject classification. Primary 41A25, 41A30, 46E30, 47A58, 47B38, 94A12.

Keywords and phrases. Sampling Kantorovich operators, Orlicz spaces, order of approximation, Lipschitz classes, irregular sampling.

Received by the editors on May 23, 2013, and in revised form on November 11, 2013.

DOI:10.1216/JIE-2014-26-3-345

Copyright (C)2014 Rocky Mountain Mathematics Consortium 


$$
\left(S_{w} f\right)(x):=\sum_{k \in \mathbf{Z}} \chi\left(w x-t_{k}\right)\left[\frac{w}{\Delta_{k}} \int_{t_{k} / w}^{t_{k+1} / w} f(u) d u\right] \quad(x \in \mathbf{R}),
$$

where $f: \mathbf{R} \rightarrow \mathbf{R}$ is a locally integrable function such that the series is convergent for every $x \in \mathbf{R}, \chi: \mathbf{R} \rightarrow \mathbf{R}$ represents a kernel function satisfying suitable properties and $\left(t_{k}\right)_{k \in \mathbf{Z}}$ is a certain increasing sequence of real numbers with $\Delta_{k}:=t_{k+1}-t_{k}>0$, for every $k \in \mathbf{Z}$. We observe that the choice of $\left(t_{k}\right)_{k \in \mathbf{Z}}$ allows us to obtain an irregular sampling scheme. The importance of studying the sampling series of the form (I) relies also on its application to the theory of Signal Processing. In particular, the multivariate versions of (I) (in linear and nonlinear cases) have applications to Image Processing (see $[\mathbf{1 9}, \mathbf{2 0}]$ ) and allows us to investigate image reconstruction.

The sampling operators (I) represent an averaged version, in the Kantorovich sense, of the generalized sampling operators introduced by Paul Leo Butzer and his school (see, e.g. , $[\mathbf{1}, \mathbf{5}, \mathbf{9}, \mathbf{1 0}, \mathbf{1 1}, \mathbf{1 2}$, $15,16,17,22,34,36,37]$ ), which consist of a generalization of the classical sampling theory based on the classical Whittaker-KotelnikovShannon sampling theorem (see, e.g., $[3,13,21,23,24,25,26,35]$ ).

One of the advantages, with respect to generalized sampling operators, given by the discrete operators (I) is that, instead of the sampling values $f(k / w)$, one has an average of $f$ on a small interval containing $k / w$ (here, instead of $k$, we have $t_{k}$ ). This situation very often occurs in Signal Processing when one cannot exactly match the node $t_{k}$ : this represents the so-called "time-jitter error." Therefore, the theory of sampling Kantorovich operators reduces time-jitter errors calculating the information in a neighborhood of a point rather than exactly at that point. Moreover, the integral form of the sampling Kantorovich operators is suitable for its study in the setting of $L^{p}$-spaces, or more generally in Orlicz spaces $L^{\varphi}(\mathbf{R})$; indeed such operators, due to their form, are continuous in $L^{\varphi}(\mathbf{R})$, and this situation, in general, does not occur for the generalized sampling operators. For the general theory of Orlicz spaces, see, e.g., $[\mathbf{2}, \mathbf{8}, \mathbf{2 7}, \mathbf{2 8}, \mathbf{2 9}, \mathbf{3 0}, \mathbf{3 2}]$.

The paper is organized as follows. Section 2 is devoted to notation and some preliminary notions concerning Orlicz spaces. In Section 3 we recall the definition of sampling Kantorovich operators and give some 
basic results, while in Section 4 we study the order of approximation in $C(\mathbf{R})$ (the set of all uniformly continuous and bounded functions) for the family $\left(S_{w} f\right)_{w>0}$. Section 5 shows the results concerning the order of approximation in the general setting of Orlicz spaces. Finally, Section 6 is devoted to the study of particular examples of kernels, such as Fejér's and B-spline kernels, to which the theory can be applied.

2. Preliminary notions. In the following, we denote by $C(\mathbf{R})$ the set of all uniformly continuous and bounded functions $f: \mathbf{R} \rightarrow \mathbf{R}$ endowed with the usual sup-norm $\|\cdot\|_{\infty}$. In order to study the rate of approximation of a family of linear operators, we recall the Zygmundtype class (Lipschitz class) in which we will work.

We define the class $\operatorname{Lip}_{\infty}(\nu), 0<\nu \leq 1$, as

$$
\begin{aligned}
\operatorname{Lip}_{\infty}(\nu): & =\left\{f \in C(\mathbf{R}):\|f(\cdot)-f(\cdot+t)\|_{\infty}\right. \\
& \left.=\mathcal{O}\left(|t|^{\nu}\right), \quad \text { as } t \rightarrow 0\right\}
\end{aligned}
$$

where, for any two functions $f, g: \mathbf{R} \rightarrow \mathbf{R}, f(t)=\mathcal{O}(g(t))$ as $t \rightarrow 0$ means that there exist constants $C, \gamma>0$ such that $|f(t)| \leq C|g(t)|$ for every $t \in[-\gamma, \gamma]([\mathbf{8}, \mathbf{3 8}])$.

We now recall some basic facts concerning Orlicz spaces.

A function $\varphi: \mathbf{R}_{0}^{+} \rightarrow \mathbf{R}_{0}^{+}$is said to be a $\varphi$-function if it satisfies the following conditions:

$(\Phi 1) \varphi$ is a non decreasing and continuous function;

$(\Phi 2) \varphi(0)=0, \varphi(u)>0$ if $u>0$ and $\lim _{u \rightarrow+\infty} \varphi(u)=+\infty$.

Let us now consider the functional $I^{\varphi}$ associated to the $\varphi$-function $\varphi$ and defined by

$$
I^{\varphi}[f]:=\int_{\mathbf{R}} \varphi(|f(x)|) d x
$$

for every $f \in M(\mathbf{R})$, i.e., for every (Lebesgue) measurable function $f: \mathbf{R} \rightarrow \mathbf{R}$. As it is well-known, $I^{\varphi}$ is a modular functional (see, e.g., $[8,30,32])$, and the Orlicz space generated by $\varphi$ is defined by

$$
L^{\varphi}(\mathbf{R}):=\left\{f \in M(\mathbf{R}): I^{\varphi}[\lambda f]<+\infty, \quad \text { for some } \lambda>0\right\} .
$$

A notion of convergence in Orlicz spaces, called modular convergence, was introduced in [31]. 
We will say that a net of functions $\left(f_{w}\right)_{w>0} \subset L^{\varphi}(\mathbf{R})$ is modularly convergent to $f \in L^{\varphi}(\mathbf{R})$, if there exists $\lambda>0$ such that

$$
I^{\varphi}\left[\lambda\left(f_{w}-f\right)\right]=\int_{\mathbf{R}} \varphi\left(\lambda\left|f_{w}(x)-f(x)\right|\right) d x \longrightarrow 0, \quad w \rightarrow+\infty .
$$

Moreover, we recall, for the sake of completeness, that in $L^{\varphi}(\mathbf{R})$ a strong notion of convergence can also be given, i.e., the Luxemburgnorm convergence, see e.g., $[\mathbf{8}, \mathbf{3 0}]$. Definition (1) induces a topology in $L^{\varphi}(\mathbf{R})$, called modular topology.

We now define by $\operatorname{Lip}_{\varphi}(\nu), 0<\nu \leq 1$, the Zygmund-type class in Orlicz spaces, as the set of all functions $f \in M(\mathbf{R})$ such that there exists $\lambda>0$ with

$$
I^{\varphi}[\lambda(f(\cdot)-f(\cdot+t))]=\int_{\mathbf{R}} \varphi(\lambda|f(x)-f(x+t)|) d x=\mathcal{O}\left(|t|^{\nu}\right),
$$

as $t \rightarrow 0$. For further results concerning Orlicz spaces, see $[\mathbf{2}, \mathbf{6}, \mathbf{8}, \mathbf{9}$, $27,28,29,30,32,33]$.

3. Sampling Kantorovich operators and some basic results. In this section, we first recall the definition of the sampling Kantorovich operators introduced in [4].

Let $\Pi=\left(t_{k}\right)_{k \in \mathbf{Z}}$ be a sequence of real numbers such that $-\infty<$ $t_{k}<t_{k+1}<+\infty$ for every $k \in \mathbf{Z}, \lim _{k \rightarrow \pm \infty} t_{k}= \pm \infty$ and there are two positive constants $\Delta, \delta$ such that $\delta \leq \Delta_{k}:=t_{k+1}-t_{k} \leq \Delta$, for every $k \in \mathbf{Z}$.

In what follows, a function $\chi: \mathbf{R} \rightarrow \mathbf{R}$ will be called a kernel if it satisfies the following properties:

- $(\chi 1) \chi \in L^{1}(\mathbf{R})$ and is bounded in a neighborhood of 0 ;

- $(\chi 2)$ for some $\mu>0$,

$\sum_{k \in \mathbf{Z}} \chi\left(w x-t_{k}\right)-1=: A_{w}(x)-1=\mathcal{O}\left(w^{-\mu}\right), \quad$ as $w \rightarrow+\infty$,

uniformly with respect to $x \in \mathbf{R}$;

- $(\chi 3)$ for some $\beta>0$,

$$
m_{\beta, \Pi}(\chi):=\sup _{u \in \mathbf{R}} \sum_{k \in \mathbf{Z}}\left|\chi\left(u-t_{k}\right)\right| \cdot\left|u-t_{k}\right|^{\beta}<+\infty ;
$$


- $(\chi 4)$ there exists $\alpha>0$ such that, for every $M>0$,

$$
\int_{|u|>M} w|\chi(w u)| d u=\mathcal{O}\left(w^{-\alpha}\right), \quad \text { as } w \rightarrow+\infty .
$$

Remark 3.1. We point out that condition $\left(\chi^{4}\right)$ is used only when we study sampling Kantorovich operators in the setting of Orlicz spaces.

We can now recall the definition of the sampling Kantorovich operators for a given kernel $\chi$. We denote by $\left(S_{w}\right)_{w>0}$ the family of operators defined by

$$
\left(S_{w} f\right)(x):=\sum_{k \in \mathbf{Z}} \chi\left(w x-t_{k}\right)\left[\frac{w}{\Delta_{k}} \int_{t_{k} / w}^{t_{k+1} / w} f(u) d u\right] \quad(x \in \mathbf{R}),
$$

where $f: \mathbf{R} \rightarrow \mathbf{R}$ is a locally integrable function such that the series is convergent for every $x \in \mathbf{R}$.

We begin giving the proof of the following lemma.

Lemma 3.2. Under the assumptions $(\chi 1)$ and $(\chi 3)$ on the kernel $\chi$, we have:

(i) $m_{0, \Pi}(\chi):=\sup _{u \in \mathbf{R}} \sum_{k \in \mathbf{Z}}\left|\chi\left(u-t_{k}\right)\right|<+\infty$;

(ii) For every $\gamma>0$

$$
\sum_{\left|w x-t_{k}\right|>\gamma w}\left|\chi\left(w x-t_{k}\right)\right|=\mathcal{O}\left(w^{-\beta}\right), \quad \text { as } w \rightarrow \infty,
$$

uniformly with respect to $x \in \mathbf{R}$, where $\beta>0$ is the constant of condition $(\chi 3)$.

Proof. For a proof of (i), see e.g., [4].

(ii) Let $\gamma>0$ be fixed. For every $x \in \mathbf{R}$ and $w>0$, we obtain:

$$
\begin{gathered}
\sum_{\left|w x-t_{k}\right|>\gamma w}\left|\chi\left(w x-t_{k}\right)\right| \leq \frac{1}{\gamma^{\beta} w^{\beta}} \\
\sum_{\left|w x-t_{k}\right|>\gamma w}\left|\chi\left(w x-t_{k}\right)\right| \cdot\left|w x-t_{k}\right|^{\beta} \leq \frac{1}{\gamma^{\beta} w^{\beta}} m_{\beta, \Pi}(\chi)<+\infty,
\end{gathered}
$$

and so the assertion follows. 


\section{Remark 3.3.}

(a) We note that, if $f \in L^{\infty}(\mathbf{R})$, using Lemma 3.2 (i), $S_{w} f$ are well-defined for every $w>0$. Indeed,

$$
\left|\left(S_{w} f\right)(x)\right| \leq m_{0, \Pi}(\chi)\|f\|_{\infty}<+\infty
$$

for every $x \in \mathbf{R}$ and $w>0$, i.e., $S_{w}: L^{\infty}(\mathbf{R}) \rightarrow L^{\infty}(\mathbf{R})$.

(b) Conditions $(\chi 3)$ is obviously fulfilled if $\chi(x)=\mathcal{O}\left(x^{-1-\beta-\varepsilon}\right)$ as $x \rightarrow \pm \infty$, for some $\varepsilon>0$. In this case, $(\chi 3)$ holds, see $[4,14]$.

(c) Instead of assuming condition $(\chi 2)$, one can directly assume the following condition:

$$
\sum_{k \in \mathbf{Z}} \chi\left(u-t_{k}\right)=1, \quad \text { for every } u \in \mathbf{R} .
$$

Clearly, if condition (3) holds, $(\chi 2)$ is fulfilled for every $\mu>0$.

Moreover, in case of the uniform sampling scheme $t_{k}=k, k \in \mathbf{Z}$, condition (3) is equivalent to:

$$
\widehat{\chi}(k):= \begin{cases}0, & k \in \mathbf{Z} \backslash\{0\}, \\ 1, & k=0,\end{cases}
$$

where $\widehat{\chi}(v):=\int_{\mathbf{R}} \chi(u) e^{-i v u} d u, v \in \mathbf{R}$, is the Fourier transform of $\chi$; see $[4,14]$.

4. Order of approximation in $C(\mathbf{R})$. We will now study the rate of approximation of the family of linear sampling Kantorovich operators (2) in $C(\mathbf{R})$. We may state the result on the order of approximation for $S_{w} f-f$ with respect to the sup-norm $\|\cdot\|_{\infty}$, with $f \in \operatorname{Lip}_{\infty}(\nu)$, $0<\nu \leq 1$.

Theorem 4.1. Let $\chi$ be a kernel and $f \in \operatorname{Lip}_{\infty}(\nu), 0<\nu \leq 1$. Then

$$
\left\|S_{w} f-f\right\|_{\infty}=\mathcal{O}\left(w^{-\epsilon}\right), \quad \text { as } w \rightarrow+\infty,
$$

where $\epsilon:=\min \{\nu, \beta, \mu\}$ and $\mu, \beta>0$ are the constants of conditions $(\chi 2)$ and $(\chi 3)$, respectively.

Proof. First, we consider the case of $\chi$ satisfying condition $(\chi 3)$ for $0<\beta \leq 1$. 
Now let $f \in \operatorname{Lip}_{\infty}(\nu), 0<\nu \leq \beta$, be fixed. By Remark 3.3 (a), $S_{w} f$ are well defined for every $w>0$. Moreover, since $f \in \operatorname{Lip}_{\infty}(\nu)$, there exist constants $C, \gamma>0$ such that

$$
\sup _{x \in \mathbf{R}}|f(x)-f(x+t)| \leq C|t|^{\nu},
$$

for every $t \in[-\gamma, \gamma]$. Now let $x \in \mathbf{R}$ be fixed. Then we can write

$$
\begin{aligned}
\left|\left(S_{w} f\right)(x)-f(x)\right| \leq & \left|\left(S_{w} f\right)(x)-f(x) A_{w}(x)\right| \\
& +\left|f(x) A_{w}(x)-f(x)\right| \\
\leq & \sum_{k \in \mathbf{Z}}\left|\chi\left(w x-t_{k}\right)\right| \frac{w}{\Delta_{k}} \int_{t_{k} / w}^{t_{k+1} / w}|f(u)-f(x)| d u \\
& +|f(x)|\left|A_{w}(x)-1\right| \\
\leq & \left(\sum_{\left|w x-t_{k}\right| \leq w \gamma / 2}+\sum_{\left|w x-t_{k}\right|>w \gamma / 2}\right)\left|\chi\left(w x-t_{k}\right)\right| \frac{w}{\Delta_{k}} \\
& \times \int_{t_{k} / w}^{t_{k+1} / w}|f(u)-f(x)| d u \\
& +\|f\|_{\infty}\left|A_{w}(x)-1\right|=: I_{1}+I_{2}+I_{3} .
\end{aligned}
$$

By putting $u=x+t$ in the integrals of $I_{1}$ and taking into account that, for every $t \in\left[t_{k} / w-x, t_{k+1} / w-x\right]$, if $\left|w x-t_{k}\right| \leq w \gamma / 2$, we have

$$
|t| \leq\left|t-t_{k} / w+x\right|+\left|t_{k} / w-x\right| \leq \frac{\Delta}{w}+\frac{\gamma}{2}<\gamma,
$$

for sufficiently large $w>0$ and then

$$
\begin{aligned}
I_{1}= & \sum_{\left|w x-t_{k}\right| \leq w \gamma / 2}\left|\chi\left(w x-t_{k}\right)\right| \frac{w}{\Delta_{k}} \\
& \times \int_{\left(t_{k} / w\right)-x}^{\left(t_{k+1} / w\right)-x}|f(x+t)-f(x)| d t \\
\leq & C \sum_{\left|w x-t_{k}\right| \leq w \gamma / 2}\left|\chi\left(w x-t_{k}\right)\right| \frac{w}{\Delta_{k}} \int_{\left(t_{k} / w\right)-x}^{\left(t_{k+1} / w\right)-x}|t|^{\nu} d t .
\end{aligned}
$$

In order to estimate (4) we introduce the following notations. We denote by $\mathcal{L}:=\left\{k \in \mathbf{Z}:\left|w x-t_{k}\right| \leq w \gamma / 2\right\}, \mathcal{L}_{1}:=\left\{k \in \mathcal{L}: t_{k} / w \geq x\right\}$ and $\mathcal{L}_{2}:=\left\{k \in \mathcal{L}: t_{k+1} / w \leq x\right\}$, with $w>0$. We can observe that $\mathcal{L}=\mathcal{L}_{1} \cup \mathcal{L}_{2} \cup \mathcal{L}_{3}$, where $\mathcal{L}_{3}:=\{\underline{k}\}$ if there exists $\underline{k} \in \mathbf{Z}$ such that 
$t_{\underline{k}} / w<x<t_{\underline{k}+1} / w$, otherwise $\mathcal{L}_{3}:=\emptyset$. In what follows, we consider only the case of $\mathcal{L}_{3}:=\{\underline{k}\}$ (the case $\mathcal{L}_{3}:=\emptyset$ is similar). Now, we rewrite the last term of (4) as follows:

$$
\begin{aligned}
& \sum_{\left|w x-t_{k}\right| \leq w \gamma / 2}\left|\chi\left(w x-t_{k}\right)\right| \frac{w}{\Delta_{k}} \int_{\left(t_{k} / w\right)-x}^{\left(t_{k+1} / w\right)-x}|t|^{\nu} d t \\
= & \left(\sum_{k \in \mathcal{L}_{1}}+\sum_{k \in \mathcal{L}_{2}}+\sum_{k \in \mathcal{L}_{3}}\right)\left|\chi\left(w x-t_{k}\right)\right| \frac{w}{\Delta_{k}} \\
& \times \int_{\left(t_{k} / w\right)-x}^{\left(t_{k+1} / w\right)-x}|t|^{\nu} d t \\
= & : J_{1}+J_{2}+J_{3} .
\end{aligned}
$$

We first estimate $J_{1}$. We have

$$
\begin{aligned}
J_{1} & \leq \sum_{k \in \mathcal{L}_{1}}\left|\chi\left(w x-t_{k}\right)\right| \sup _{t \in\left[\left(t_{k} / w\right)-x,\left(t_{k+1} / w\right)-x\right]}|t|^{\nu} \\
& \leq \sum_{k \in \mathcal{L}_{1}}\left|\chi\left(w x-t_{k}\right)\right|\left|t_{k+1} / w-x\right|^{\nu} \\
& =\frac{1}{w^{\nu}} \sum_{k \in \mathcal{L}_{1}}\left|\chi\left(w x-t_{k}\right)\right|\left|t_{k}+\Delta_{k}-w x\right|^{\nu},
\end{aligned}
$$

for every $w>0$. Now, since $0<\nu \leq \beta \leq 1$, we have that $|\cdot|^{\nu}$ is concave, and then subadditive, so we obtain

$$
\begin{aligned}
J_{1} \leq & w^{-\nu} \sum_{k \in \mathcal{L}_{1}}\left|\chi\left(w x-t_{k}\right)\right|\left[\left|t_{k}-w x\right|^{\nu}+\Delta_{k}^{\nu}\right] \\
\leq & w^{-\nu} \sum_{k \in \mathcal{L}_{1}}\left|\chi\left(w x-t_{k}\right)\right|\left|w x-t_{k}\right|^{\nu} \\
& +w^{-\nu} \Delta^{\nu} \sum_{k \in \mathcal{L}_{1}}\left|\chi\left(w x-t_{k}\right)\right| .
\end{aligned}
$$

We estimate $J_{2}$. We have

$$
\begin{aligned}
J_{2} & \leq \sum_{k \in \mathcal{L}_{2}}\left|\chi\left(w x-t_{k}\right)\right| \sup _{t \in\left[\left(t_{k} / w\right)-x,\left(t_{k+1} / w\right)-x\right]}|t|^{\nu} \\
& \leq \sum_{k \in \mathcal{L}_{2}}\left|\chi\left(w x-t_{k}\right)\right|\left|t_{k} / w-x\right|^{\nu}
\end{aligned}
$$




$$
=w^{-\nu} \sum_{k \in \mathcal{L}_{2}}\left|\chi\left(w x-t_{k}\right)\right|\left|w x-t_{k}\right|^{\nu},
$$

for every $w>0$. Finally, we estimate $J_{3}$. Using again the subadditivity of $|\cdot|^{\nu}$, we obtain

$$
\begin{aligned}
J_{3} \leq & \left|\chi\left(w x-t_{\underline{k}}\right)\right|_{t \in\left[\left(t_{\underline{k}} / w\right)-x,\left(t_{\underline{k}+1} / w\right)-x\right]}|t|^{\nu} \\
\leq & \left|\chi\left(w x-t_{\underline{k}}\right)\right| \max \left\{\left|t_{\underline{k}} / w-x\right|^{\nu},\left|t_{\underline{k}+1} / w-x\right|^{\nu}\right\} \\
\leq & \frac{1}{w^{\nu}}\left|\chi\left(w x-t_{\underline{k}}\right)\right|\left[\left|t_{\underline{k}}-w x\right|^{\nu}+\Delta^{\nu}\right] \\
= & w^{-\nu}\left|\chi\left(w x-t_{\underline{k}}\right)\right|\left|w x-t_{\underline{k}}\right|^{\nu} \\
& +w^{-\nu} \Delta^{\nu}\left|\chi\left(w x-t_{\underline{k}}\right)\right|,
\end{aligned}
$$

for every $w>0$. Then, by the above estimates, we can state that

$$
\begin{aligned}
J_{1}+J_{2}+J_{3} \leq & w^{-\nu} \sum_{k \in \mathcal{L}}\left|\chi\left(w x-t_{k}\right)\right|\left|w x-t_{k}\right|^{\nu} \\
& +w^{-\nu} \Delta^{\nu} \sum_{k \in \mathcal{L}_{1} \cup \mathcal{L}_{3}}\left|\chi\left(w x-t_{k}\right)\right| \\
\leq & w^{-\nu}\left[m_{\nu, \Pi}(\chi)+\Delta^{\nu} m_{0, \Pi}(\chi)\right] .
\end{aligned}
$$

Now, since if $m_{\beta, \Pi}(\chi)<+\infty$ then $m_{\nu, \Pi}(\chi)<+\infty$ for every $0<\nu \leq \beta$, by condition $(\chi 3)$ and Lemma 3.2 (i) we have

$$
\begin{aligned}
J_{1}+J_{2}+J_{3} & \leq w^{-\nu}\left[m_{\nu, \Pi}(\chi)+\Delta^{\nu} m_{0, \Pi}(\chi)\right] \\
& =: w^{-\nu} M<+\infty,
\end{aligned}
$$

for every fixed $w>0$. Then, by the above inequality and the estimate in (4), we obtain that

$$
I_{1}=\mathcal{O}\left(w^{-\nu}\right), \quad \text { as } w \rightarrow+\infty,
$$

uniformly with respect to $x \in \mathbf{R}$. Further,

$$
I_{2} \leq 2\|f\|_{\infty} \sum_{\left|w x-t_{k}\right|>w \gamma / 2}\left|\chi\left(w x-t_{k}\right)\right|=\mathcal{O}\left(w^{-\beta}\right), \quad \text { as } w \rightarrow \infty,
$$

uniformly with respect to $x \in \mathbf{R}$, as a consequence of Lemma 3.2 (ii). Finally, from condition $(\chi 2)$ we obtain that $I_{3}=\mathcal{O}\left(w^{-\mu}\right)$, as $w \rightarrow+\infty$, uniformly with respect to $x \in \mathbf{R}$. Thus, we have shown that

$$
\left|\left(S_{w} f\right)(x)-f(x)\right| \leq I_{1}+I_{2}+I_{3}
$$




$$
=\mathcal{O}\left(w^{-\nu}\right)+\mathcal{O}\left(w^{-\beta}\right)+\mathcal{O}\left(w^{-\mu}\right), \quad \text { as } w \rightarrow \infty,
$$

uniformly with respect to $x \in \mathbf{R}$ and, therefore, we finally obtain that

$$
\left\|S_{w} f-f\right\|_{\infty}=\mathcal{O}\left(w^{-\epsilon}\right), \quad \text { as } w \rightarrow+\infty,
$$

where $\epsilon:=\min \{\nu, \beta, \mu\}$.

Let us now consider the case $f \in \operatorname{Lip}_{\infty}(\nu)$, with $\beta \leq \nu \leq 1$ fixed. It is easy to observe that $\operatorname{Lip}_{\infty}(\nu) \subseteq \operatorname{Lip}_{\infty}(\beta)$, so this can be reduced to the above case, from which we obtain that

$$
\left\|S_{w} f-f\right\|_{\infty}=\mathcal{O}\left(w^{-\epsilon}\right), \quad \text { as } w \rightarrow+\infty,
$$

where $\epsilon:=\min \{\beta, \mu\}=\min \{\nu, \beta, \mu\}$, since $\nu \geq \beta$.

Finally, we study the case of $\chi$ satisfying condition $(\chi 3)$ for $\beta>1$. As noted above, $m_{\beta, \Pi}(\chi)<+\infty$ implies $m_{1, \Pi}(\chi)<+\infty$, which means the kernel satisfies condition $(\chi 3)$ also for $\beta=1$, and so this can be reduced to the previous case. This completes the proof.

5. Order of approximation in Orlicz spaces. We will now study the order of approximation for the sampling Kantorovich operators in the general setting of Orlicz spaces $L^{\varphi}(\mathbf{R})$, for the $\varphi$ convex. First of all, we recall a modular continuity property for our operators $S_{w}$.

Theorem 5.1. Let $\chi$ be a kernel. For every $f \in L^{\varphi}(\mathbf{R})$, the following holds:

$$
I^{\varphi}\left[\lambda S_{w} f\right] \leq \frac{\|\chi\|_{1}}{\delta m_{0, \Pi}(\chi)} I^{\varphi}\left[\lambda m_{0, \Pi}(\chi) f\right] \quad(\lambda>0),
$$

for every $w>0$. In particular, $S_{w} f \in L^{\varphi}(\mathbf{R})$ whenever $f \in L^{\varphi}(\mathbf{R})$.

For a proof of Theorem 5.1, see [4].

Now, we establish the following result which gives a degree of approximation for the sampling Kantorovich operators in Orlicz spaces.

Theorem 5.2. Let $\chi$ be a kernel. Moreover, let $f \in L^{\varphi}(\mathbf{R}) \cap \operatorname{Lip}_{\varphi}(\nu)$, $0<\nu \leq 1$, be fixed. Suppose, in addition, that there exist $\theta$ and $\gamma>0$ such that

$$
\int_{|t| \leq \gamma} w|\chi(w t)||t|^{\nu} d t=\mathcal{O}\left(w^{-\theta}\right), \quad \text { as } w \rightarrow+\infty .
$$


Then there exists $\lambda>0$ such that

$$
I^{\varphi}\left[\lambda\left(S_{w} f-f\right)\right]=\mathcal{O}\left(w^{-\epsilon}\right), \quad \text { as } w \longrightarrow+\infty,
$$

with $\epsilon:=\min \{\theta, \nu, \mu, \alpha\}$, where $\mu, \alpha>0$ are the constants of conditions $(\chi 2)$ and $(\chi 4)$, respectively.

Proof. First of all, by the assumption $f \in L^{\varphi}(\mathbf{R}) \cap \operatorname{Lip}_{\varphi}(\nu), 0<\nu \leq$ 1 , we have that there exist $\lambda_{1}, \lambda_{2}>0$ such that $I^{\varphi}\left[\lambda_{1} f\right]<+\infty$, and

$$
I^{\varphi}\left[\lambda_{2}(f(\cdot)-f(\cdot+t))\right]=\mathcal{O}\left(|t|^{\nu}\right), \quad \text { as } t \rightarrow 0,
$$

i.e., there exist $M_{1}$ and $\bar{\gamma}>0$ such that

$$
I^{\varphi}\left[\lambda_{2}(f(\cdot)-f(\cdot+t))\right] \leq M_{1}|t|^{\nu},
$$

for every $|t| \leq \bar{\gamma}$. Now, by the properties of the convex modular functional $I^{\varphi}$, for $\lambda>0$, we can write:

$$
\begin{aligned}
I^{\varphi}\left[\lambda\left(S_{w} f-f\right)\right]= & \int_{\mathbf{R}} \varphi\left(\lambda\left|\left(S_{w} f\right)(x)-f(x)\right|\right) d x \\
\leq & \frac{1}{3}\left\{\int _ { \mathbf { R } } \varphi \left(3 \lambda \mid\left(S_{w} f\right)(x)\right.\right. \\
& \left.-\sum_{k \in \mathbf{Z}} \chi\left(w x-t_{k}\right) \frac{w}{\Delta_{k}} \int_{t_{k} / w}^{t_{k+1} / w} f\left(u+x-\frac{t_{k}}{w}\right) d u \mid\right) d x \\
& +\int_{\mathbf{R}} \varphi\left(3 \lambda \mid \sum_{k \in \mathbf{Z}} \chi\left(w x-t_{k}\right) \frac{w}{\Delta_{k}}\right. \\
& \left.\times \int_{t_{k} / w}^{t_{k+1} / w} f\left(u+x-\frac{t_{k}}{w}\right) d u-f(x) A_{w}(x) \mid\right) d x \\
& \left.+\int_{\mathbf{R}} \varphi\left(3 \lambda\left|f(x) A_{w}(x)-f(x)\right|\right) d x\right\} \\
= & \frac{1}{3}\left\{J_{1}+J_{2}+J_{3}\right\} .
\end{aligned}
$$

First, we estimate $J_{1}$. Applying Jensen's inequality and the FubiniTonelli theorem,

$$
J_{1}=\int_{\mathbf{R}} \varphi\left(3 \lambda \mid\left(S_{w} f\right)(x)\right.
$$




$$
\begin{aligned}
& \left.-\sum_{k \in \mathbf{Z}} \chi\left(w x-t_{k}\right) \frac{w}{\Delta_{k}} \int_{t_{k} / w}^{t_{k+1} / w} f\left(u+x-\frac{t_{k}}{w}\right) d u \mid\right) d x \\
\leq & \frac{1}{m_{0, \Pi}(\chi)} \int_{\mathbf{R}} \sum_{k \in \mathbf{Z}}\left|\chi\left(w x-t_{k}\right)\right| \varphi\left(3 \lambda m_{0, \Pi}(\chi) \frac{w}{\Delta_{k}}\right. \\
& \left.\times \int_{t_{k} / w}^{t_{k+1} / w}\left|f(u)-f\left(u+x-\frac{t_{k}}{w}\right)\right| d u\right) d x \\
\leq & \frac{1}{m_{0, \Pi}(\chi)} \sum_{k \in \mathbf{Z}} \int_{\mathbf{R}}\left|\chi\left(w x-t_{k}\right)\right| \varphi\left(3 \lambda m_{0, \Pi}(\chi) \frac{w}{\Delta_{k}}\right. \\
& \left.\times \int_{t_{k} / w}^{t_{k+1} / w}\left|f(u)-f\left(u+x-\frac{t_{k}}{w}\right)\right| d u\right) d x .
\end{aligned}
$$

Putting $t=x-t_{k} / w$ and applying the Fubini-Tonelli theorem and Jensen's inequality again, we may write

$$
\begin{aligned}
J_{1} \leq & \frac{1}{m_{0, \Pi}(\chi)} \int_{\mathbf{R}}|\chi(w t)| \sum_{k \in \mathbf{Z}} \varphi\left(3 \lambda m_{0, \Pi}(\chi) \frac{w}{\Delta_{k}}\right. \\
& \left.\times \int_{t_{k} / w}^{t_{k+1} / w}|f(u)-f(u+t)| d u\right) d t \\
\leq & \frac{1}{m_{0, \Pi}(\chi)} \int_{\mathbf{R}}|\chi(w t)|\left\{\sum_{k \in \mathbf{Z}} \frac{w}{\Delta_{k}}\right. \\
& \left.\times \int_{t_{k} / w}^{t_{k+1} / w} \varphi\left(3 \lambda m_{0, \Pi}(\chi)|f(u)-f(u+t)|\right) d u\right\} d t \\
\leq & \frac{1}{m_{0, \Pi}(\chi) \delta} \int_{\mathbf{R}} w|\chi(w t)| \\
& \left\{\sum_{k \in \mathbf{Z}} \int_{t_{k} / w}^{t_{k+1} / w} \varphi\left(3 \lambda m_{0, \Pi}(\chi)|f(u)-f(u+t)|\right) d u\right\} d t \\
= & \frac{1}{m_{0, \Pi}(\chi) \delta} \int_{\mathbf{R}} w|\chi(w t)| \\
& \times\left\{\int_{\mathbf{R}} \varphi\left(3 \lambda m_{0, \Pi}(\chi)|f(u)-f(u+t)|\right) d u\right\} d t \\
= & \frac{1}{m_{0, \Pi}(\chi) \delta}\left\{\int_{|t| \leq \widetilde{\gamma}} w|\chi(w t)|\right.
\end{aligned}
$$




$$
\begin{aligned}
& \times\left(\int_{\mathbf{R}} \varphi\left(3 \lambda m_{0, \Pi}(\chi)|f(u)-f(u+t)|\right) d u\right) d t \\
& \left.+\int_{|t|>\widetilde{\gamma}} w|\chi(w t)|\left(\int_{\mathbf{R}} \varphi\left(3 \lambda m_{0, \Pi}(\chi)|f(u)-f(u+t)|\right) d u\right) d t\right\} \\
= & \frac{1}{m_{0, \Pi}(\chi) \delta}\left\{J_{1,1}+J_{1,2}\right\},
\end{aligned}
$$

with $\widetilde{\gamma}:=\min \{\gamma, \bar{\gamma}\}$, where $\gamma>0$ is the constant of condition (6). Now, we fix $\lambda>0$ such that:

$$
\lambda \leq \min \left\{\frac{\lambda_{1}}{3 M_{2}}, \frac{\lambda_{1}}{6 m_{0, \Pi}(\chi)}, \frac{\lambda_{2}}{3 m_{0, \Pi}(\chi)}, \frac{\lambda_{2} \delta}{6 \Delta}\right\},
$$

where $M_{2}>0$ is obtained from condition $(\chi 2)$, i.e., $M_{2}$ is such that

$$
\left|\sum_{k \in \mathbf{Z}} \chi\left(w x-t_{k}\right)-1\right| \leq M_{2} w^{-\mu}
$$

uniformly with respect to $x \in \mathbf{R}$ and for sufficiently large $w>0$. Since $f \in \operatorname{Lip}_{\varphi}(\nu)$ and by condition (6), we obtain:

$$
\begin{aligned}
J_{1,1} & \leq \int_{|t| \leq \widetilde{\gamma}} w|\chi(w t)|\left[\int_{\mathbf{R}} \varphi\left(\lambda_{2}|f(u)-f(u+t)|\right) d u\right] d t \\
& \leq M_{1} \int_{|t| \leq \widetilde{\gamma}} w|\chi(w t)||t|^{\nu} d t \\
& =\mathcal{O}\left(w^{-\theta}\right), \quad \text { as } w \rightarrow+\infty .
\end{aligned}
$$

For $J_{1,2}$, by the convexity of $\varphi$, we have

$$
\begin{aligned}
J_{1,2} \leq \int_{|t|>\widetilde{\gamma}} w|\chi(w t)| \frac{1}{2}\left[\int_{\mathbf{R}} \varphi\right. & \left(6 \lambda m_{0, \Pi}(\chi)|f(u)|\right) d u \\
& \left.+\int_{\mathbf{R}} \varphi\left(6 \lambda m_{0, \Pi}(\chi)|f(u+t)|\right) d u\right] d t
\end{aligned}
$$

and noting that

$$
\int_{\mathbf{R}} \varphi\left(6 \lambda m_{0, \Pi}(\chi)|f(u+t)|\right) d u=\int_{\mathbf{R}} \varphi\left(6 \lambda m_{0, \Pi}(\chi)|f(u)|\right) d u,
$$

for every $t \in \mathbf{R}$, we obtain 


$$
\begin{aligned}
& J_{1,2} \leq \int_{|t|>\widetilde{\gamma}} w|\chi(w t)| \int_{\mathbf{R}} \varphi\left(6 \lambda m_{0, \Pi}(\chi)|f(u)|\right) d u \\
&=I^{\varphi}\left[6 \lambda m_{0, \Pi}(\chi) f\right] \int_{|t|>\widetilde{\gamma}} w|\chi(w t)| d t
\end{aligned}
$$

Therefore, by the above inequality, the choice of $\lambda$ and condition $\left(\chi^{4}\right)$, it follows immediately that

$$
J_{1,2}=\mathcal{O}\left(w^{-\alpha}\right), \quad \text { as } w \rightarrow+\infty .
$$

Now, we estimate $J_{2}$. Putting $t=u-t_{k} / w$ and by the convexity of $\varphi$ we have

$$
\begin{aligned}
J_{2} \leq & \int_{\mathbf{R}} \varphi\left(3 \lambda \mid \sum_{k \in \mathbf{Z}} \chi\left(w x-t_{k}\right)\right. \\
& \left.\times\left[\frac{w}{\Delta_{k}} \int_{t_{k} / w}^{t_{k+1} / w} f\left(u+x-\frac{t_{k}}{w}\right) d u-f(x)\right] \mid\right) d x \\
= & \int_{\mathbf{R}} \varphi\left(3 \lambda\left|\sum_{k \in \mathbf{Z}} \chi\left(w x-t_{k}\right)\left[\frac{w}{\Delta_{k}} \int_{0}^{\Delta_{k} / w} f(x+t) d t-f(x)\right]\right|\right) d x \\
= & \int_{\mathbf{R}} \varphi\left(3 \lambda\left|\sum_{k \in \mathbf{Z}} \chi\left(w x-t_{k}\right) \frac{w}{\Delta_{k}} \int_{0}^{\Delta_{k} / w}[f(x+t)-f(x)] d t\right|\right) d x \\
\leq & \int_{\mathbf{R}} \varphi\left(3 \lambda\left|\sum_{k \in \mathbf{Z}} \chi\left(w x-t_{k}\right) \frac{w}{\delta} \int_{0}^{\Delta_{k} / w}\right| f(x+t)-f(x)|d t|\right) d x \\
\leq & \int_{\mathbf{R}} \varphi\left(3 \lambda\left|\sum_{k \in \mathbf{Z}} \chi\left(w x-t_{k}\right) \frac{w}{\delta} \int_{0}^{\Delta / w}\right| f(x+t)-f(x)|d t|\right) d x \\
\leq & \frac{1}{2}\left\{\int_{\mathbf{R}} \varphi\left(6 \lambda\left|\sum_{k \in \mathbf{Z}} \chi\left(w x-t_{k}\right)-1\right| \frac{w}{\delta} \int_{0}^{\Delta / w}|f(x+t)-f(x)| d t\right) d x\right. \\
& \left.+\int_{\mathbf{R}} \varphi\left(6 \lambda \frac{w}{\delta} \int_{0}^{\Delta / w}|f(x+t)-f(x)| d t\right) d x\right\} .
\end{aligned}
$$

By condition $(\chi 2)$, the monotonicity of $\varphi$, Jensen's inequality and Fubini-Tonelli theorem, we obtain, for sufficiently large $w$,

$$
J_{2} \leq \frac{1}{2}\left\{\int_{\mathbf{R}} \varphi\left(6 \lambda M_{2} w^{-\mu} \frac{w}{\delta} \int_{0}^{\Delta / w}|f(x+t)-f(x)| d t\right) d x\right.
$$




$$
\begin{aligned}
& \left.+\int_{\mathbf{R}} \varphi\left(6 \lambda \frac{w}{\delta} \int_{0}^{\Delta / w}|f(x+t)-f(x)| d t\right) d x\right\} \\
\leq & \int_{\mathbf{R}} \varphi\left(6 \lambda \frac{w}{\delta} \int_{0}^{\Delta / w}|f(x+t)-f(x)| d t\right) d x \\
\leq & \int_{\mathbf{R}} \frac{w}{\Delta}\left\{\int_{0}^{\Delta / w} \varphi\left(6 \lambda \frac{\Delta}{\delta}|f(x+t)-f(x)|\right) d t\right\} d x \\
= & \frac{w}{\Delta} \int_{0}^{\Delta / w}\left\{\int_{\mathbf{R}} \varphi\left(6 \lambda \frac{\Delta}{\delta}|f(x+t)-f(x)|\right) d x\right\} d t,
\end{aligned}
$$

where in the above inequalities the constant $6 \lambda M_{2} w^{-\mu} \leq 6 \lambda$ for sufficiently large $w>0$. Now, since $f \in \operatorname{Lip}_{\varphi}(\nu)$, by the choice of $\lambda$, and by the change of variable $t=u / w$, we have that

$$
J_{2} \leq \frac{M_{1}}{\Delta} w \int_{0}^{\Delta / w}|t|^{\nu} d t=\frac{M_{1}}{\Delta} w^{-\nu} \int_{0}^{\Delta}|u|^{\nu} d u=: C w^{-\nu},
$$

for sufficiently large $w>0$, i.e., $J_{2}=\mathcal{O}\left(w^{-\nu}\right)$, as $w \rightarrow+\infty$.

Finally, we estimate $J_{3}$. By condition $(\chi 2)$, the convexity of $\varphi$ and for sufficiently large $w>0$, we have

$$
\begin{aligned}
J_{3} & \leq \int_{\mathbf{R}} \varphi\left(3 \lambda|f(x)|\left|A_{w}(x)-1\right|\right) d x \\
& \leq \int_{\mathbf{R}} \varphi\left(3 \lambda M_{2} w^{-\mu}|f(x)|\right) d x \\
& \leq w^{-\mu} I^{\varphi}\left[3 M_{2} \lambda f\right] \leq w^{-\mu} I^{\varphi}\left[\lambda_{1} f\right] \\
& <+\infty .
\end{aligned}
$$

In conclusion, combining all the above estimates, we obtain

$$
I^{\varphi}\left[\lambda\left(S_{w} f-f\right)\right]=\mathcal{O}\left(w^{-\epsilon}\right), \quad \text { as } w \rightarrow+\infty,
$$

where $\epsilon:=\min \{\theta, \nu, \mu, \alpha\}$.

Note that condition (6) is satisfied when, for instance, the kernel $\chi$ is with compact support, with supp $\chi \subset[-B, B], B>0$. Indeed,

$$
\int_{|t| \leq \gamma} w|\chi(w t)||t|^{\nu} d t \leq \int_{|u| \leq B}|\chi(u)|\left|\frac{u}{w}\right|^{\nu} d u=: K w^{-\nu},
$$


for sufficiently large $w>0$, i.e., $\theta=\nu$. Moreover, in the above case, $\chi$ satisfies condition $(\chi 4)$ for every $\alpha>0$. Hence, we obtain the following.

Corollary 5.3. Let $\chi$ be a kernel with compact support. Moreover, let $f \in L^{\varphi}(\mathbf{R}) \cap \operatorname{Lip}_{\varphi}(\nu), 0<\nu \leq 1$. Then, there exists $\lambda>0$ such that

$$
I^{\varphi}\left[\lambda\left(S_{w} f-f\right)\right]=\mathcal{O}\left(w^{-\epsilon}\right), \quad \text { as } w \rightarrow+\infty,
$$

with $\epsilon:=\min \{\nu, \mu\}$, where $\mu>0$ is the constant of condition $(\chi 2)$.

If the kernel $\chi$ has not compact support, we may require that the absolute moment

$$
m_{\nu}(\chi):=\int_{\mathbf{R}}|\chi(u)||u|^{\nu} d u<+\infty,
$$

for some $0<\nu \leq 1$. In this case, for every $\gamma>0$ we have

$$
\int_{|t| \leq \gamma} w|\chi(w t)||t|^{\nu} d t \leq \int_{|u| \leq \gamma w}|\chi(u)|\left|\frac{u}{w}\right|^{\nu} d u \leq m_{\nu}(\chi) w^{-\nu},
$$

for every $w>0$, which shows that (6) holds for $\theta=\nu$. Thus, we obtain the following.

Corollary 5.4. Let $\chi$ be a kernel with $m_{\nu}(\chi)<+\infty$, for some $0<\nu \leq 1$. Moreover, let $f \in L^{\varphi}(\mathbf{R}) \cap \operatorname{Lip}_{\varphi}(\nu)$ be fixed. Then, there exists $\lambda>0$ such that

$$
I^{\varphi}\left[\lambda\left(S_{w} f-f\right)\right]=\mathcal{O}\left(w^{-\epsilon}\right), \quad \text { as } w \rightarrow+\infty,
$$

with $\epsilon:=\min \{\nu, \mu, \alpha\}$, where $\mu>0$ and $\alpha>0$ are the constants of conditions $(\chi 2)$ and $(\chi 4)$, respectively.

Remark 5.5. Convex $\varphi$-functions generating remarkable examples of Orlicz spaces where the theory of sampling Kantorovich operators is valid are: $\varphi_{p}(u):=u^{p}, 1 \leq p<\infty, \varphi_{\alpha, \beta}:=u^{\alpha} \log ^{\beta}(u+e)$, for $\alpha \geq 1$, $\beta>0$ and $\varphi_{\gamma}(u)=e^{u^{\gamma}}-1$, for $\gamma>0, u \geq 0$. It is well known that $\varphi_{p}$ generates the $L^{p}(\mathbf{R})$-space and the corresponding convex modular functional is given by $I^{\varphi_{p}}[f]:=\|f\|_{p}^{p}$. While $\varphi_{\alpha, \beta}$ and $\varphi_{\gamma}$ generate the $L^{\alpha} \log ^{\beta} L$-spaces (or Zygmund spaces), largely used in the theory of partial differential equations, and the exponential spaces respectively, 


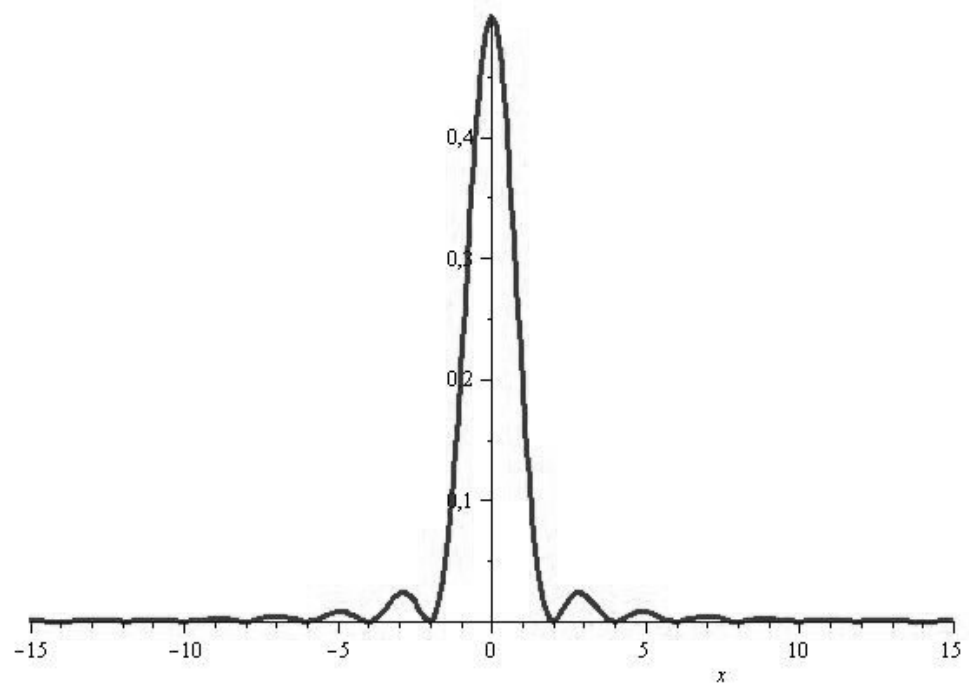

Figure 1. The Fejér's kernel $F$.

used for embedding theorems between Sobolev spaces. The convex modular functionals corresponding to $\varphi_{\alpha, \beta}$ and $\varphi_{\gamma}$ are

$$
I^{\varphi_{\alpha, \beta}}[f]:=\int_{\mathbf{R}}|f(x)|^{\alpha} \log ^{\beta}(e+|f(x)|) d x, \quad(f \in M(\mathbf{R})),
$$

and

$$
I^{\varphi_{\gamma}}[f]:=\int_{\mathbf{R}}\left(e^{|f(x)|^{\gamma}}-1\right) d x, \quad(f \in M(\mathbf{R})),
$$

respectively.

6. Applications to special kernels. As a first example, we consider the sampling Kantorovich operators based upon the Fejér's kernel (see Figure 1), of the form

$$
F(x):=\frac{1}{2} \operatorname{sinc}^{2}\left(\frac{x}{2}\right), \quad(x \in \mathbf{R}),
$$


where the sinc-function and its Fourier transform are given by

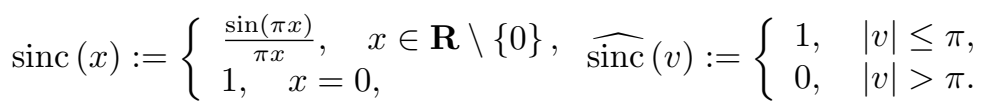

Clearly, $F$ is bounded, belongs to $L^{1}(\mathbf{R})$ and satisfies the moment conditions $(\chi 3)$ for $\beta=1$ in view of Remark $3.3(\mathrm{~b})$, see $[\mathbf{4}, \mathbf{1 4}]$. Moreover, for every $M>0$

$$
\int_{|u|>M} w F(w u) d u \leq \frac{2}{\pi^{2}} w^{-1} \int_{|u|>M} \frac{1}{u^{2}} d u=: K w^{-1},
$$

for every $w>0$, then condition $(\chi 4)$ holds for $\alpha=1$. Furthermore, taking into account that the Fourier transform of $F$ is given by (see [14])

$$
\widehat{F}(v):=\left\{\begin{array}{l}
1-|v / \pi|, \quad|v| \leq \pi, \\
0, \quad|v|>\pi,
\end{array}\right.
$$

we obtain by Remark 3.3 (c) that, for the case of uniform-spaced sampling $t_{k}=k, k \in \mathbf{Z}$, condition $(\chi 2)$ is fulfilled for every $\mu>0$. Finally, in [14] is proved that, the Fejér's kernel has the absolute moments $m_{\nu}(F)<+\infty$, for every $0<\nu \leq 1$. Then, the sampling Kantorovich operators based on the Fejér's kernel now take the form

$$
\left(S_{w}^{F} f\right)(x)=\frac{1}{2} \sum_{k \in \mathbf{Z}}\left[w \int_{k / w}^{(k+1) / w} f(u) d u\right] \operatorname{sinc}^{2}\left(\frac{w x-k}{2}\right), \quad(x \in \mathbf{R}),
$$

for every $w>0$, where $f: \mathbf{R} \rightarrow \mathbf{R}$ is a locally integrable function such that the above series is convergent for every $x \in \mathbf{R}$. For $S_{w}^{F} f$, from Theorem 4.1, Theorem 5.1 and Corollary 5.4, we can obtain, respectively, the following.

Corollary 6.1. Let $f \in \operatorname{Lip}_{\infty}(\nu)$, with $0<\nu \leq 1$. Then

$$
\left\|S_{w}^{F} f-f\right\|_{\infty}=\mathcal{O}\left(w^{-\nu}\right), \quad \text { as } w \rightarrow+\infty .
$$

In case of Orlicz spaces, for every $f \in L^{\varphi}(\mathbf{R})$, the following holds:

$$
I^{\varphi}\left[\lambda S_{w}^{F} f\right] \leq \frac{1}{\delta} I^{\varphi}[\lambda f]
$$

for some $\lambda>0$ and for every $w>0$. In particular, $S_{w}^{F} f \in L^{\varphi}(\mathbf{R})$ whenever $f \in L^{\varphi}(\mathbf{R})$. 
Moreover, for any $f \in L^{\varphi}(\mathbf{R}) \cap \operatorname{Lip}_{\varphi}(\nu), 0<\nu \leq 1$, there exists $\lambda>0$ such that

$$
I^{\varphi}\left[\lambda\left(S_{w}^{F} f-f\right)\right]=\mathcal{O}\left(w^{-\nu}\right), \quad \text { as } w \rightarrow+\infty .
$$

We observe that, since the Fejér's kernel has unbounded support, for the reconstruction of a given signal $f$ by means of $S_{w}^{F} f$, we need an infinite number of mean values $w \int_{k / w}^{(k+1) / w} f(u) d u$ in order to evaluate our operators at any fixed point $x \in \mathbf{R}$. Therefore, for a practical application of the theory of sampling Kantorovich operators based upon kernel having unbounded support, the sampling series must be truncated, and this produces truncation errors.

In order to avoid this problem, we can take into consideration kernels with compact support. In this case, the infinite sampling series computed at any $x \in \mathbf{R}$ reduces to a finite one. Remarkable examples of such kernels are given by the well-known B-splines of order $n \in \mathbf{N}$ (see Figure 2 for the B-spline of order 3), defined by

$$
M_{n}(x):=\frac{1}{(n-1) !} \sum_{i=0}^{n}(-1)^{i}\left(\begin{array}{c}
n \\
i
\end{array}\right)\left(\frac{n}{2}+x-i\right)_{+}^{n-1},
$$

where the function $(x)_{+}:=\max \{x, 0\}$ denotes the positive part of $x \in \mathbf{R}$ (see $[4,14,39]$ ).

We have that the Fourier transform of $M_{n}$ is given by

$$
\widehat{M_{n}}(v):=\operatorname{sinc}^{n}\left(\frac{v}{2 \pi}\right), \quad(v \in \mathbf{R}),
$$

and then, if we consider the case of the uniform sampling scheme, condition $(\chi 2)$ is satisfied for every $\mu>0$, by Remark 3.3 (c). Clearly, $M_{n}$ are bounded on $\mathbf{R}$, with compact support [ $\left.-n / 2, n / 2\right]$, and hence $M_{n} \in L^{1}(\mathbf{R})$, for all $n \in \mathbf{N}$. Moreover, it is easy to deduce that conditions $(\chi 3)$ and $(\chi 4)$ are fulfilled for every $\beta>0$ and $\alpha>0$. Then, the sampling Kantorovich operators based on the B-spline kernel of order $n$ now take the form

$$
\left(S_{w}^{M_{n}} f\right)(x)=\sum_{k \in \mathbf{Z}}\left[w \int_{k / w}^{(k+1) / w} f(u) d u\right] M_{n}(w x-k),(x \in \mathbf{R}),
$$




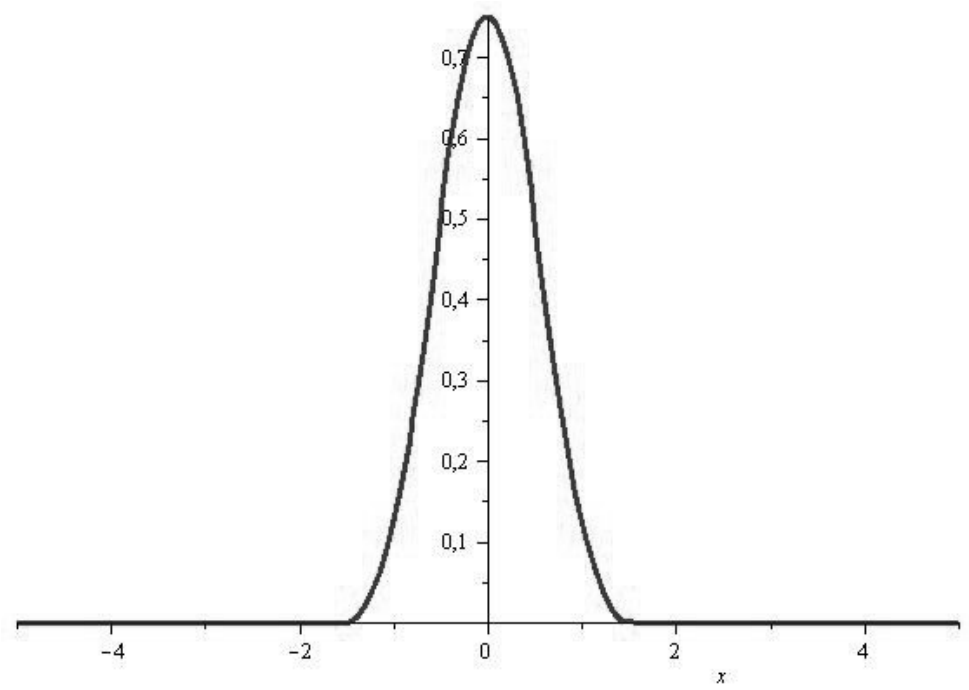

Figure 2. The B-spline of order 3.

for every $w>0$, where $f: \mathbf{R} \rightarrow \mathbf{R}$ is a locally integrable function such that the above series is convergent for every $x \in \mathbf{R}$. From Theorem 4.1 and Corollary 5.3, we obtain the following.

Corollary 6.2. Let $f \in \operatorname{Lip}_{\infty}(\nu)$, with $0<\nu \leq 1$. Then

$$
\left\|S_{w}^{M_{n}} f-f\right\|_{\infty}=\mathcal{O}\left(w^{-\nu}\right), \quad \text { as } w \rightarrow+\infty .
$$

In case of Orlicz spaces, for every $f \in L^{\varphi}(\mathbf{R})$, the following holds:

$$
I^{\varphi}\left[\lambda S_{w}^{M_{n}} f\right] \leq \frac{1}{\delta} I^{\varphi}[\lambda f],
$$

for some $\lambda>0$ and for every $w>0$. In particular, $S_{w}^{M_{n}} f \in L^{\varphi}(\mathbf{R})$ whenever $f \in L^{\varphi}(\mathbf{R})$.

Moreover, for any $f \in L^{\varphi}(\mathbf{R}) \cap \operatorname{Lip}_{\varphi}(\nu), 0<\nu \leq 1$, there exists a $\lambda>0$ such that

$$
I^{\varphi}\left[\lambda\left(S_{w}^{M_{n}} f-f\right)\right]=\mathcal{O}\left(w^{-\nu}\right), \quad \text { as } w \rightarrow+\infty .
$$


For other useful examples of kernels, see e.g., $[4,8,14,38]$.

We now consider the situations above described in some special case of Orlicz spaces. As a first example we study the case $L^{\varphi}(\mathbf{R})=$ $L^{\alpha} \log ^{\beta}(\mathbf{R})$ with $\alpha \geq 1$ and $\beta>0$. The Zygmund spaces are examples of Orlicz spaces for which the corresponding $\varphi$-functions $\varphi_{\alpha, \beta}(u)=$ $u^{\alpha} \log ^{\beta}(u+e), u \geq 0$, satisfy the well-known $\Delta_{2}$-condition (see, e.g., $[8,30,31])$. We write the following corollaries for the operators $S_{w}^{\chi}$, with $\chi=F$ or $\chi=M_{n}$.

Corollary 6.3. For every $f \in L^{\alpha} \log ^{\beta}(\mathbf{R})$, with $\alpha \geq 1$ and $\beta>0$, the following holds:

$$
\begin{aligned}
\int_{\mathbf{R}}\left|\left(S_{w}^{\chi} f\right)(x)\right|^{\alpha} \log ^{\beta}\left(e+\lambda\left|\left(S_{w}^{\chi} f\right)(x)\right|\right) d x & \\
& \leq \frac{1}{\delta} \int_{\mathbf{R}}|f(x)|^{\alpha} \log ^{\beta}(e+\lambda|f(x)|) d x,
\end{aligned}
$$

for some $\lambda>0$ and for every $w>0$. In particular, $S_{w}^{\chi} f \in L^{\alpha} \log ^{\beta}(\mathbf{R})$ whenever $f \in L^{\alpha} \log ^{\beta}(\mathbf{R})$.

Moreover, for any $f \in L^{\alpha} \log ^{\beta}(\mathbf{R}) \cap \operatorname{Lip}_{\varphi_{\alpha, \beta}}(\nu), 0<\nu \leq 1$, there exists $a \lambda>0$ such that

$$
\int_{\mathbf{R}}\left|\left(S_{w}^{\chi} f\right)(x)\right|^{\alpha} \log ^{\beta}\left(e+\lambda\left|\left(S_{w}^{\chi} f\right)(x)\right|\right) d x=\mathcal{O}\left(w^{-\nu}\right) \text {, as } w \rightarrow+\infty .
$$

A further important class of Orlicz spaces, to which the theory of sampling Kantorovich operators can be applied, is that one generated by the $\varphi$-functions $\varphi_{p}(u)=u^{p}, p \geq 1, u \geq 0$. Obviously, $\varphi_{p}(u)$ satisfy the $\Delta_{2}$-condition and the corresponding Orlicz spaces are the wellknown $L^{p}$-spaces. Corollary 6.3 can be easily formulated also in the $L^{p}$ setting.

Finally, important examples of Orlicz spaces generated by $\varphi$-functions which do not satisfy the $\Delta_{2}$-condition are given, e.g., by the exponential spaces generated by the $\varphi$-functions $\varphi_{\gamma}(u)=e^{u^{\gamma}}-1, \gamma>0$ and $u \geq 0$. In the latter setting, we point out the following corollary concerning the order of approximation for the sampling Kantorovich operators $S_{w}^{\chi}$, with $\chi=F$ or $\chi=M_{n}$. 
Corollary 6.4. For every $f \in L^{\varphi_{\gamma}}(\mathbf{R}), \gamma>0$, the following holds:

$$
\int_{\mathbf{R}}\left(e^{\left|\lambda\left(S_{w}^{\chi} f\right)(x)\right|^{\gamma}}-1\right) d x \leq \frac{1}{\delta} \int_{\mathbf{R}}\left(e^{|\lambda f(x)|^{\gamma}}-1\right) d x,
$$

for some $\lambda>0$ and for every $w>0$. In particular, $S_{w}^{\chi} f \in L^{\varphi_{\gamma}}(\mathbf{R})$ whenever $f \in L^{\varphi_{\gamma}}(\mathbf{R})$.

Moreover, for any $f \in L^{\varphi_{\gamma}}(\mathbf{R}) \cap \operatorname{Lip}_{\varphi_{\gamma}}(\nu), 0<\nu \leq 1$, there exists a $\lambda>0$ such that

$$
\int_{\mathbf{R}}\left(e^{\left|\lambda\left(S_{w}^{\chi} f\right)(x)\right|^{\gamma}}-1\right) d x=\mathcal{O}\left(w^{-\nu}\right), \quad \text { as } w \rightarrow+\infty .
$$

\section{REFERENCES}

1. L. Angeloni and G. Vinti, Rate of approximation for nonlinear integral operators with applications to signal processing, Diff. Integ. Equat. 18 (2005), 855890.

2. J. Appell, The importance of being Orlicz, in Orlicz centenary volume, Banach Center Publ., Polish Acad. Sci. 64, 2004.

3. C. Bardaro, P.L. Butzer, R.L. Stens and G. Vinti, Approximation of the Whittaker Sampling Series in terms of an average modulus of smoothness covering discontinuous signals, J. Math. Anal. Appl. 316 (2006), 269-306.

4. Kantorovich-type generalized sampling series in the setting of Orlicz spaces, Sampl. Theor. Signal Image Process. 6 (2007), 29-52.

5. _ Prediction by samples from the past with error estimates covering discontinuous signals, IEEE Trans. Infor. Theor. 56 (2010), 614-633.

6. C. Bardaro and I. Mantellini, Modular approximation by sequences of nonlinear integral operators in Musielak-Orlicz Spaces, Att. Mat. Fis. Univ. Modena 46 (1998), 403-425.

7. On convergence properties for a class of Kantorovich discrete operators, Numer. Funct. Anal. Optim. 33 (2012), 374-396.

8. C. Bardaro, J. Musielak and G. Vinti, Nonlinear integral operators and applications, De Gruyter Ser. Nonlin. Anal. Appl. 9, 2003.

9. C. Bardaro and G. Vinti, Some inclusion theorems for Orlicz and MusielakOrlicz type spaces, Ann. Mat. Pura Appl. 168 (1995), 189-203.

10. , A general approach to the convergence theorems of generalized sampling series, Appl. Anal. 64 (1997), 203-217.

11. , Uniform convergence and rate of approximation for a nonlinear version of the generalized sampling operator, Results Math. 34 (1998), 224-240.

12. An abstract approach to sampling type operators inspired by the work of P.L. Butzer-Part I-Linear operators, Samp. Theor. Signal Image Proc. 2 (2003), 271-296. 
13. L. Bezuglaya and V. Katsnelson, The sampling theorem for functions with limited multi-band spectrum I, Z. Anal. Anwend. 12 (1993), 511-534.

14. P.L. Butzer and R.J. Nessel, Fourier analysis and approximation I, Academic Press, New York, 1971.

15. P.L. Butzer, S. Ries and R.L. Stens, Approximation of continuous and discontinuous functions by generalized sampling series, J. Approx. Theor. 50 (1987), 25-39.

16. P.L. Butzer and R.L. Stens, Sampling theory for not necessarily band-limited functions: A historical overview, SIAM Rev. 34 (1992), 40-53.

17. Linear prediction by samples from the past, in Advanced topics in Shannon sampling and interpolation theory, R.J. Marks, II, ed., Springer-Verlag, New York, 1993.

18. F. Cluni, D. Costarelli, A.M. Minotti and G. Vinti, Multivariate sampling Kantorovich operators: Approximation and applications to civil engineering, in EURASIP, Proc. SampTA 2013, 10th Int. Conf. Sampl. Theor. Appl., 400-403.

19. D. Costarelli and G. Vinti, Approximation by multivariate generalized sampling Kantorovich operators in the setting of Orlicz spaces, Boll. U.M.I. 4 (2011), $445-468$.

20. Approximation by nonlinear multivariate sampling Kantorovich type operators and applications to image processing, Num. Funct. Anal. Optim. 34 (2013), 819-844.

21. M.M. Dodson and A.M. Silva, Fourier analysis and the sampling theorem, Proc. Irish Acad. 86 (1985), 81-108.

22. C. Donnini and G. Vinti, Approximation by means of Kantorovich generalized sampling operators in Musielak-Orlicz spaces, PanAmer. Math. J.18 (2008), $1-18$.

23. J.R. Higgins, Five short stories about the cardinal series, Bull. Amer. Math. Soc. 12 (1985), 45-89.

24. _ Sampling theory in Fourier and signal analysis: Foundations, Oxford University Press, Oxford, 1996.

25. J.R. Higgins and R.L. Stens, Sampling theory in Fourier and signal analysis: Advanced topics, Oxford Science Publications, Oxford University Press, Oxford, 1999.

26. A.J. Jerry, The Shannon sampling-Its various extensions and applications: A tutorial review, Proc. IEEE 65 (1977), 1565-1596.

27. W.M. Kozlowski, Modular function spaces, Pure Appl. Math. Marcel Dekker, New York, 1988.

28. M.A. Krasnosel'skiǐ and Ya.B. Rutickiř, Convex functions and Orlicz spaces, P. Noordhoff Ltd., Groningen, The Netherlands, 1961.

29. L. Maligranda, Orlicz spaces and interpolation, Sem. Mat. IMECC, Campinas, 1989.

30. J. Musielak, Orlicz spaces and modular spaces, Lec. Notes in Math. 1034, Springer-Verlag, New York, 1983. 
31. J. Musielak and W. Orlicz, On modular spaces, Stud. Math. 28 (1959), 49-65.

32. M.M. Rao and Z.D. Ren, Theory of Orlicz spaces, Pure Appl. Math., Marcel Dekker, Inc., New York, 1991.

33. _ Applications of Orlicz spaces, Mono. Text. Pure Appl. Math. 250, Marcel Dekker, Inc., New York, 2002.

34. S. Ries and R.L. Stens, Approximation by generalized sampling series, in Constructive theory of functions'84, Sofia, 1984.

35. C.E. Shannon, Communication in the presence of noise, Proc. I.R.E. 37 (1949), 10-21.

36. C. Vinti, A survey on recent results of the mathematical seminar in Perugia, inspired by the work of Professor P.L. Butzer, Result. Math. 34 (1998), 32-55.

37. G. Vinti, A general approximation result for nonlinear integral operators and applications to signal processing, Appl. Anal. 79 (2001), 217-238.

38. Approximation in Orlicz spaces for linear integral operators and applications, Rend. Circ. Mat. Palermo 76 (2005), 103-127.

39. G. Vinti and L. Zampogni, Approximation by means of nonlinear Kantorovich sampling type operators in Orlicz spaces, J. Approx. Theor. 161 (2009), $511-528$.

40. , A unifying approach to convergence of linear sampling type operators in Orlicz spaces, Adv. Differ. Equat. 16 (2011), 573-600.

Dipartimento di Matematica e Fisica, Sezione di Matematica, Università degli Studi Roma Tre, 1, Largo S. Leonardo Murialdo, 00146 Rome, Italy Email address: costarel@mat.uniroma3.it

Dipartimento di Matematica e Informatica, Università degli Studi di Perugia, 1, Via Vanvitelli, 06123, Perugia, Italy

Email address: gianluca.vinti@unipg.it 